\title{
Neue Option für Volumenaufbau
}

\author{
New Options for Volumisation
}

Autoren

Institute
T. Walker ${ }^{1}$, C. BayerI ${ }^{2}$

Private Hautarztpraxis Dermatologie, Allergologie, Ästhetische Dermatologie, Ludwigshafen

2 Klinik für Dermatologie und Allergologie, Wilhelm-Fresenius-Klinik, Wiesbaden
Bibliografie

DOI http://dx.doi.org/ $10.1055 / \mathrm{s}-0030-1255609$

Online-Publikation: 10. 8. 2010

Akt Dermatol 2010; 36:

361-365 @ Georg Thieme

Verlag KG Stuttgart · New York ISSN 0340-2541

Korrespondenzadresse

Dr. Torsten Walker

Private Hautarztpraxis

Kurfürstenstraße 32

67061 Ludwigshafen

Torsten_Walker@gmx.de

\section{Zusammenfassung \\ $\nabla$}

Hintergrund: $\quad$ Novabel $^{\circledR}$ ist der erste Filler auf Basis von Meeresalgen. Wir haben das Alginat-Gel zum Volumenaufbau der Hände eingesetzt und dokumentiert.

Material und Methoden: Es nahmen 10 Patienten an der Dokumentation teil. Die Effektivität wurde mit der Global Aesthetic Improvement Scale (GAIS) bestimmt. Verträglichkeit, Schmerzempfinden, Applikationsfreundlichkeit, Injektionsdruck wurden dokumentiert.

\section{Hintergrund und Fragestellung \\ $\checkmark$}

Eine der bewährten Methoden zur minimal-invasiven Behandlung von Volumen und Konturdefekten ist die Anwendung sogenannter „dermaler Filler“. Diese Füllsubstanzen werden zur Faltenbehandlung, Lippenaugmentation, Volumengebung und Gesichtskonturierung eingesetzt.

Novabel $^{\circledR}$ stellt eine neue Kategorie unter den Dermalfillern dar. Novabel ${ }^{\circledR}$ ist die erste Füllsubstanz auf der Basis von natürlich gewonnenen Meeresalgen der Spezies Laminaria pallida. Das gewonnene Alginat besteht aus Zuckermolekülen, welche in einem speziellen biotechnischen Prozess zu einem viskoelastischen, kohäsiven Gel umgewandelt werden. Bei diesem Vernetzungsprozess entstehen runde 3D-Mikrosphären, die gleichmäßig in Größe und Anordnung dichtest gepackt sind. So bildet sich eine „HomogeneousOrbital-Matrix“ (HOM-Struktur ${ }^{\mathrm{TM}}$ ) aus, in der Millionen von Alginat-Sphären flexibel angeordnet sind, aber dennoch ihre Form behalten. Insofern zeichnet sich dieses Gel durch flexible und zugleich strukturstabile Eigenschaften aus und eignet sich insbesondere für ein effektives Konturieren und zum gezielten Volumenaufbau.
Ergebnisse: Sofort nach Injektion zeigte sich bei 80\% der Patienten eine Verbesserung auf der GAIS, bei $20 \%$ war der Zustand „sehr verbessert“. Die Verträglichkeit im Anschluss an die Injektion wurde bei allen Patienten als „exzellent“ bezeichnet. Reaktionen im Injektionsbereich traten bei 33,3\% der Patienten auf. Das Alginat-Gel ließ sich leicht injizieren und war schmerzarm in der Anwendung.

Schlussfolgerung: Novabel ${ }^{\circledR}$ stellt eine neue Option für den Volumenaufbau in bisher wenig behandelten Arealen dar.

Im Gegensatz zu konventionellen Hyaluronsäuren, die über Enzyme (Hyaluronidasen) abgebaut werden, gibt es im menschlichen Körper kein alginatspezifisches abbauendes Enzym. Novabel ${ }^{\circledR}$ wird daher nur über den langsam verlaufenden Prozess der Hydrolyse abgebaut und führt so zu länger anhaltenden kosmetischen Ergebnissen [1]. Alginate wirken nicht irritierend, sind gut verträglich und nicht immunstimulierend. In Form eines Füllmaterials ermöglicht Novabel ${ }^{\circledR}$ insbesondere die Behandlung von anspruchsvollen Arealen wie z.B. Infraorbitalbereich, aber auch Jochbögen, Wangen, Unterkieferkontur, Dekolleté und Händen.

Das Wissen um die Eigenschaften der Filler, deren spezielle Anwendungstechniken und geeignete Indikationsbereiche sind Voraussetzung für überzeugende Behandlungsresultate bei gleichzeitig geringer Komplikationsrate. Die Effektivität, Wirkdauer und Verträglichkeit von Novabel $^{\circledR}$ wird zur Zeit in einer randomisierten multizentrischen klinischen Studie mit 154 Patienten in der Behandlung der moderaten bis starken Nasolabialfalte untersucht [1]. Der vorliegende Bericht ergänzt dieses Wissen durch Erfahrungen aus der niedergelassenen Praxis beim Volumenaufbau der Hände. 


\section{Studiendesign und Untersuchungsmethoden}

$\nabla$

\section{Patienten}

Es handelte sich um einen unkontrollierten monozentrischen prospektiven Erfahrungsbericht, der in unserer niedergelassenen Praxis der Fachrichtungen Dermatologie, Allergologie und Ästhetische Dermatologie in Ludwigshafen von August bis Dezember 2009 erhoben wurde. Insgesamt nahmen 10 Patienten (> 18 Jahre) an der Dokumentation teil. Ausgeschlossen waren Patienten mit

- bekannter Allergie gegen einen der Bestandteile,

- Neigung zu Keloiden oder Granulomen,

- einer entzündeten oder infizierten Haut,

- klinisch relevanter Blutungsneigung oder Antikoagulationstherapie,

- Schwangere und Stillende.

Die Erhebungsdauer betrug ca. 3 Monate pro Patient. Die Daten wurden vor der Behandlung, direkt nach der Injektion (Soforteffekt), nach ca. 2 - 4 Wochen und nach ca. 12 Wochen dokumentiert.

Jeder in den Erfahrungsbericht aufgenommene Patient wurde ärztlich über die Bedeutung und Durchführung der Novabe ${ }^{\circledR}{ }^{\circledR}$ Behandlung aufgeklärt und erteilte seine schriftliche Einwilligung zur Teilnahme und zur Veröffentlichung der Fotos.

\section{Material und Anwendung}

In der vorliegenden Erhebung dokumentierten wir den Volumenaufbau der Hände mit Novabel ${ }^{\circledR}$ (Merz Pharmaceuticals $\mathrm{GmbH}$, Deutschland, z.Zt. nicht erhältlich), einem quervernetzten Alginat-Gel (12 mg/ml Ringer-Lösung, Natriumhydrogencarbonatpuffer), abgefüllt in einer $1 \mathrm{ml}$-Einmalspritze.

Die Anwendung erfolgte gemäß der Gebrauchsinformation. Demnach wird Novabel ${ }^{\circledR}$ mit der beiliegenden 30 G $1 / 2$-Nadel in die tiefe Dermis bzw. obere Subkutis injiziert. Dies kann mittels der multiplen Punkttechnik, der linearen Tunneltechnik, der Sandwich- oder Fächertechnik geschehen. Speziell für den Handrücken wird die Zelt- (Tenting-) oder Bolus-Technik empfohlen. Eine Überkorrektur und Dosierung $>6 \mathrm{ml}$ pro Behandlungszyklus sollte nicht erfolgen.

\section{Tenting-Technik (O Abb. 1)}

- Je Injektionspunkt die Dermis mit zwei Fingern zeltartig anheben (Licht scheint durch).

- Unter die hochgezogene Hautfalte zwischen den Bändern in Tunnel- oder Depot-Technik injizieren, die Falte loslassen.

- Unter Druckmassage das Material verteilen und modellieren.

\section{Bolus-Technik (0 Abb. 2)}

- Bolusinjektion entlang der ausgedünnten Sehnenfächer am Übergang Dermis-Subkutis.

- Material zwischen den Sehnenfächern auf dem Handrücken verteilen und ausmassieren.

\section{Auswertungsparameter}

Vor Behandlungsbeginn wurde der Ausprägungsgrad des Volumendefizits anhand einer 3-stufigen Skala (leicht, mittel, schwer) festgestellt.

Das kosmetische Ergebnis wurde im Vergleich zum Ausgangszustand anhand der 5 Punkte umfassenden Global Aesthetic Improvement Scale (GAIS) bestimmt: $0=$ schlechter, $1=$ unverändert, $2=$ verbessert, $3=$ sehr verbessert, $4=$ sehr stark verbessert [3]. Die Erhebung des GAIS fand an allen drei Kontrollterminen der Nachbeobachtungszeit statt.
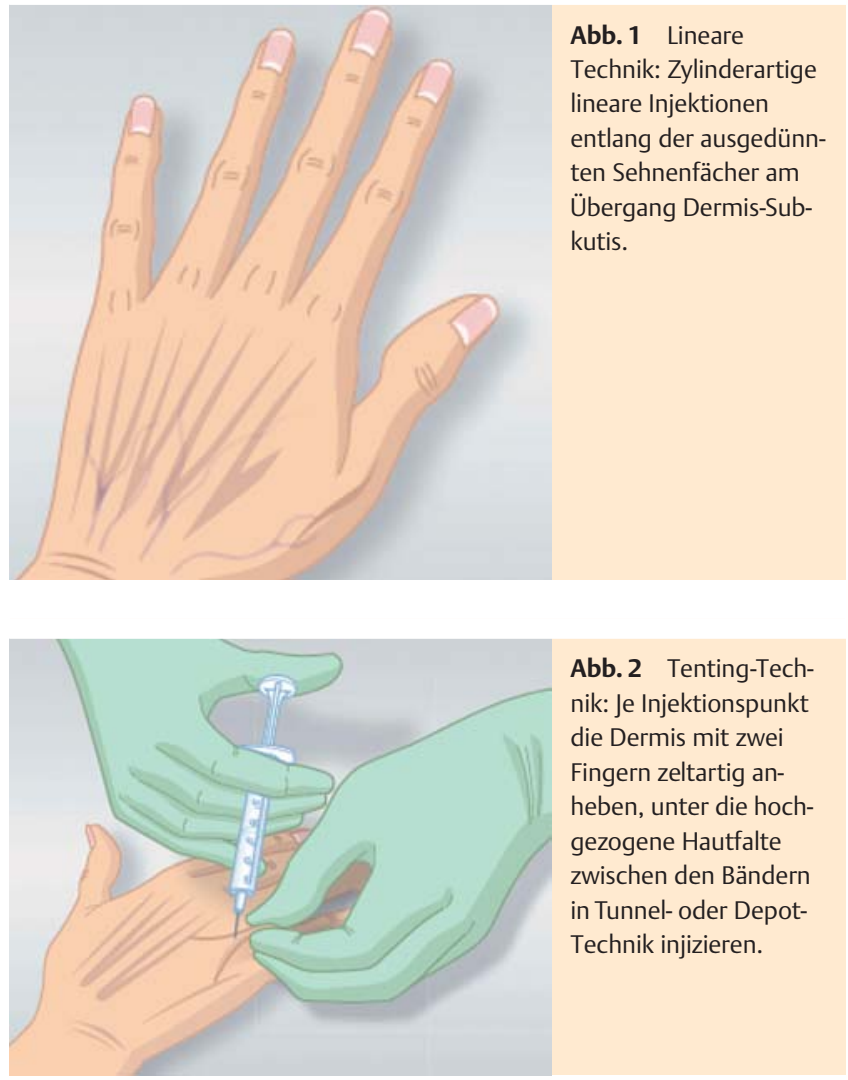

Um die Handhabung zu bewerten, wurden mögliche Applikationsprobleme definiert und deren Häufigkeit (nie, eher selten, manchmal, häufiger, sehr häufig) dokumentiert. Ebenso wurde der Injektionsdruck anhand einer 5-stufigen Skala erfasst ( 1 = niedrig, 5 = hoch) und einer bewertenden Einschätzung inklusive deren Begründung unterzogen.

Zu jedem Nachbeobachtungstermin wurde die Verträglichkeit in Form einer globalen Beurteilung und die Reaktionen im Implantatbereich dokumentiert. Während und nach der Injektion gaben die Patienten ihr Schmerzempfinden auf der numerischen Rating Skala von $0=$ keine Schmerzen bis $10=$ sehr starke Schmerzen zu Protokoll.

\section{Statistik}

Die statistische Auswertung wurde von einem unabhängigen Statistik-Institut durchgeführt. Die Datenverarbeitung und die biometrische Auswertung wurden mithilfe des Programms „SPSS 15.0 für Windows“ vorgenommen.

Gemäß dem offenen Studiendesign erfolgte die statistische Auswertung in erster Linie mithilfe von deskriptiven Methoden. Für kontinuierliche Daten wurden die üblichen Kenngrößen berechnet (Anzahl n, arithmetisches Mittel, Standardabweichung, Minimum, Maximum). Qualitative Daten wurden mit absoluten und relativen Häufigkeiten beschrieben.

\section{Ergebnisse}

\section{Demografische Patientendaten}

Insgesamt nahmen 10 Patienten an der Erhebung teil, davon 9 Frauen und 1 Mann. Im Mittel betrug das Alter der Patienten 57 Jahre und variierte zwischen 45 und 73 Jahren. 


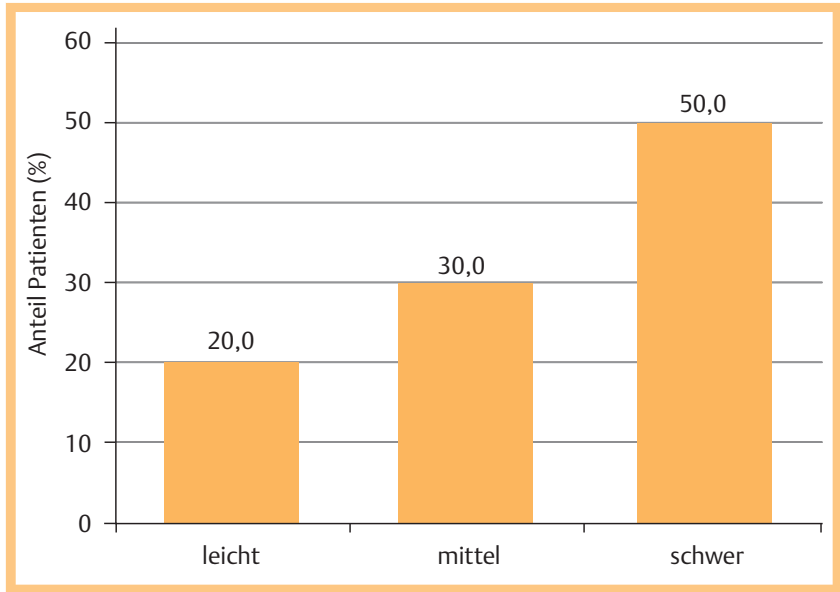

Abb. 3 Ausprägung des Volumendefizits.

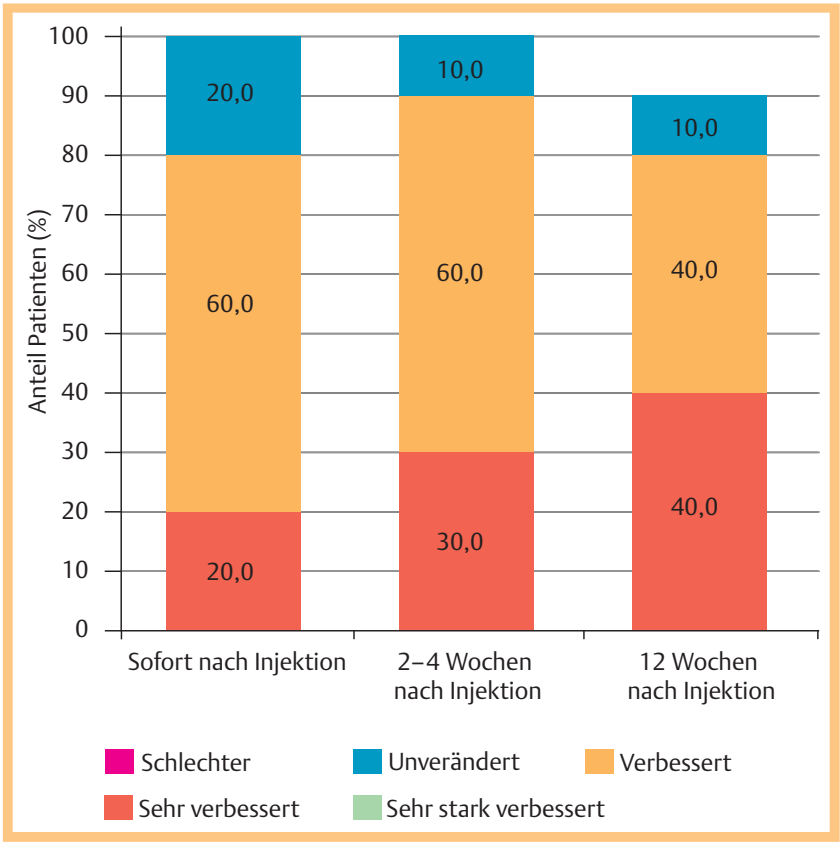

Abb. 4 Verbesserung gemäß der GAIS.

\section{Basisdaten}

Die Hälfte der Patienten wies ein schweres Volumendefizit auf, $30 \%$ hatten einen mittleren und 20\% einen leichten Ausprägungsgrad $(\bullet$ Abb. 3$)$.

Novabel ${ }^{\circledR}$ wurde in einer mittleren Menge von $3,6 \mathrm{ml}$ injiziert (Minimum 2,0 ml; Maximum $5 \mathrm{ml}$ ). Eine lokale Anästhesie in Form einer anästhesierenden Creme war in keiner der Anwendungen erforderlich.

Die Applikation erfolgte mit der Bolus-Technik bzw. der ZeltTechnik. Bei der Mehrzahl der Patienten (50\%) kamen beide Techniken zur Anwendung, 30\% wurden ausschließlich mit der Bolus-Technik injiziert, $10 \%$ ausschließlich mit der Zelt-Technik.

\section{Effektivität/Wirksamkeit}

Sofort nach der Injektion war bei 80\% der Patienten eine Verbesserung gegenüber dem Ausgangszustand feststellbar, bei $20 \%$ wurde der Zustand sogar als "sehr verbessert“ angegeben (৫ Abb. 4).

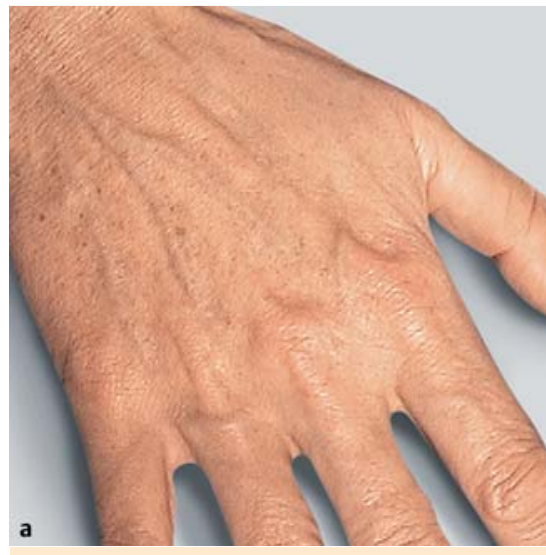

Abb. 5 a Patientin vor Behandlung. b Patientin 14 Tage nach Behandlung.

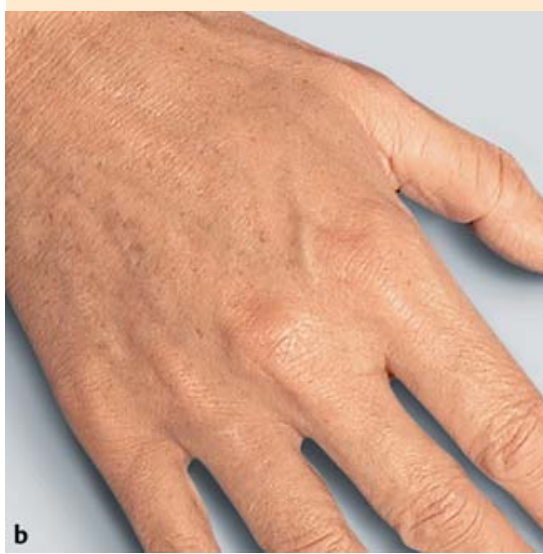

Nur 2 Patienten waren „unverändert“ geblieben. Die Einschätzung dieses Behandlungserfolgs steigerte sich während der 3monatigen Beobachtung weiter. Nach 12 Wochen waren die Hände bei jeweils $40 \%$ der Patienten „sehr verbessert“ bzw. „verbessert“. In keinem Fall trat eine Verschlechterung ein und nur ein Patient blieb „unverändert“.

In der Mehrzahl der Fälle wurden die Behandlungsergebnisse fotografisch dokumentiert. Ein Beispiel zeigt $\bullet$ Abb. 5 a und b.

\section{Sicherheit und Verträglichkeit}

Novabel ${ }^{\circledR}$ erwies sich als ausgezeichnet verträglich. Lediglich bei 3 Patienten traten temporäre Reaktionen im Implantatbereich auf:

- ein Patient mit Rötung an der Einstichstelle im Anschluss an die Injektion und massiver Schwellung nach 4 Wochen, welche bei einem Aufenthalt in großer Höhe (Hochgebirge) auftrat. Nach Rückkehr aus dem Gebirge war die Schwellung komplett rückläufig und nicht wieder auftretend. Möglicherweise führte die Veränderung der Druckverhältnisse zu diesem Effekt.

- ein Patient mit leichter Verhärtung nach 2-4 Wochen, die sich bis zum nächsten Kontrolltermin zurückgebildet hatte, und

- ein Patient mit bläulicher Verfärbung nach Injektion, welche aber eher im Sinne einer Akrozyanose zu werten war.

Schmerzen traten nur bei der Injektion selbst auf. Sie waren mit einem Mittelwert von 1,6 Punkten auf der numerischen Rating Skala (NRS) ausschließlich leichter Natur und nach der Injektion bei allen Patienten bereits abgeklungen.

Dementsprechend war im globalen Urteil die Verträglichkeit im direkten Anschluss an die Injektion bei allen Patienten als „exzellent“ zu bezeichnen. Diese gute Beurteilung bestätigte sich im Wesentlichen auch bei den späteren Nachbeobachtungsterminen 
(nach 2-4 Wochen: 40\% „exzellent“, 50\% „sehr gut“, bei einem Patienten „mittelmäßig“; nach 12 Wochen: 10\% „exzellent“, $70 \%$ „sehr gut“, bei einem Patienten „mittelmäßig“).

\section{Handhabung und Applikationsfreundlichkeit}

Die Handhabung war problemlos.

In allen Fällen wurde der Injektionsdruck als niedrig eingestuft und wegen der damit verbundenen leichten Dosierbarkeit als ideal empfunden.

\section{Diskussion}

$\nabla$

Das neue Alginat-Gel Novabel ${ }^{\circledR}$ erweitert seit Kurzem das Spektrum ästhetischer minimal-invasiver Behandlungsoptionen. Es besteht aus natürlichen Polysacchariden, die aus Meeresalgen gewonnen werden. Diese bilden in einem patentierten Verfahren runde Sphären, welche dichtest gepackt sind. Der Sphärenzwischenraum ist mit Ringerlösung ausgefüllt. Diese HomogeneousOrbital-Matrix-(HOM-)Struktur ${ }^{\mathrm{TM}}$ bildet ein Gel, das die notwendige Stabilität für einen Volumenaufbau mitbringt und gleichzeitig so niedrig viskös ist, dass es leicht und weitgehend schmerzfrei zu injizieren ist. Vor diesem Hintergrund haben wir das Alginat-Gel in unserer Praxis bei 10 Patienten zum Volumenaufbau der Hände eingesetzt und sein kosmetisches Ergebnis, seine Verträglichkeit und seine Handhabung dokumentiert.

Bis auf einen Patienten, bei dem der ästhetische Zustand unverändert blieb, sprachen alle gut auf die Behandlung mit Novabel ${ }^{\circledR}$ an. Die Injektion führte bei $80 \%$ der Patienten zu einer sofortigen Verbesserung, die sich im Laufe des Beobachtungszeitraums kontinuierlich weiter steigerte. Nach 12 Wochen waren gemäß GAISScore die Hände bei jeweils $40 \%$ der Patienten sehr verbessert bzw. verbessert. Ein Patient fehlte beim letzten Dokumentationstermin. Im Gegensatz zu unserem Behandlungsverlauf zeigte sich in einer klinischen Studie mit 154 Patienten bei der Behandlung der moderaten bis starken Nasolabialfalte der volle Effekt sofort nach der Injektion [1].

Idealerweise sollte ein Füllmaterial keine signifikante Immunantwort, allergische oder Entzündungsreaktion auslösen, leicht injizierbar, wenig schmerzhaft für den Patienten sein, am Injektionsort verbleiben und einen möglichst lang anhaltenden Fülleffekt haben [4]. Doch jeder Fremdstoff, der unserem Körper verabreicht wird, kann Abwehrmechanismen aktivieren und immunologische Reaktionen hervorrufen. So kommt es nach der Injektion von Kollagen- und Hyaluronsäure-Fillern nicht selten zu leichten Entzündungsreaktionen [2,5-7].

Unser Erfahrungsbericht mit der neuen Füllsubstanz zeigt eine sehr gute Verträglichkeit von Novabel ${ }^{\circledR}$. Lediglich bei 3 Patienten $(33,3 \%)$ traten temporäre Reaktionen im Implantatbereich auf. Hierbei handelte es sich um transiente Rötungen, Schwellungen, minimale Verhärtung oder Verfärbung. Die Behandlung konnte ohne jegliche Anästhesie durchgeführt werden. Es traten nur leichte Schmerzen (im Mittel 1,6 Punkte auf der NRS), und zwar unmittelbar während der Injektion auf. Danach waren alle Patienten wieder schmerzfrei. Auf Basis dieser Daten war im globalen Urteil die Verträglichkeit im Anschluss an die Injektion bei allen Patienten als „exzellent“ zu bezeichnen. Diese gute Beurteilung konnte im Wesentlichen auch bei den späteren Nachbeobachtungsterminen bestätigt werden.

Diese Beobachtungen stehen im Einklang mit bisherigen Daten zur initialen Verträglichkeit von Novabel $^{\circledR}$, die Rötungen mit einer Häufigkeit von 28,5\%, Schwellungen mit 8,4\% und Schmerzen mit 1,3\% ausweisen [1]. Im Vergleich hierzu werden z. B. bei Hyaluronsäure-Fillern Rötungen, Schwellungen und Schmerzen bei bis zu 90\% der Patienten angegeben [6]. Insofern verwundert es nicht, dass auch in einer klinischen Studie mit 154 Patienten die Prüfärzte über den gesamten Beobachtungszeitraum von 18 Monaten die Verträglichkeit von Novabel ${ }^{\circledR}$ bei mehr als 95\% der Patienten durchgängig mit „sehr gut“ bzw. „gut“ bewerteten [1]. Neben der guten kosmetischen Wirkung und der Verträglichkeit verdienen insbesondere auch Handhabung und Applikationsfreundlichkeit von Novabel ${ }^{\circledR}$ ausdrückliche Beachtung. In allen Fällen wurde der Injektionsdruck als niedrig eingestuft - ein wichtiger Faktor für leichte Dosierbarkeit und schmerzarme Behandlung. Diese Injektionsfreundlichkeit wird auf die niedrige Viskosität und hohe Elastizität des Materials zurückgeführt. Der Injektionsdruck von nur 8 Newton liegt weit unterhalb desjenigen von Hyaluronsäure-Fillern [8]. Deshalb empfiehlt es sich, den Daumen nach der Implantation bzw. bereits beim Herausziehen der Nadel aus dem Gewebe von dem Stempel zu nehmen, um die Platzierung in den oberen Gewebeschichten auszuschließen. Ausschlaggebend für den niedrigen Injektionsdruck sind die gleichmäßig runden Alginat-Sphären, die mit $150 \pm 30 \mu \mathrm{m}$ so klein sind, dass sie bei der Injektion problemlos die Nadel passieren. Insofern lässt sich aus unserer Erfahrung heraus Novabel ${ }^{\circledR}$ leichter und schmerzloser injizieren im Vergleich zu beispielsweise biphasischen Hyaluronsäure-Fillern.

\section{Fazit für die Praxis}

$\nabla$

Das Alginat-Gel Novabel ${ }^{\circledR}$ zeigte insgesamt gute kosmetische Ergebnisse beim Volumenaufbau der Hände und erwies sich als sehr gut verträglich. Besonders hervorzuheben ist seine leichte Injizierbarkeit und schmerzarme Anwendung. Durch diese Eigenschaften eignet sich Novabel ${ }^{\circledR}$ insbesondere auch für den Volumenaufbau anspruchsvoller Areale und kann damit neue Potenziale erschließen, die bisher wenig genutzt wurden.

Interessenkonflikt: Der korrespondierende Autor weist auf folgende Beziehungen hin: Die Erhebung wurde gesponsert durch Merz Pharmaceuticals GmbH.

\section{Abstract}

\section{New Options for Volumisation}

Background: Novabel $^{\circledR}$ is the first dermal filler extracted from seaweed. We employed this alginate gel for hand augmentation and documentation.

Material and Methods: A total of 10 patients received injections. Efficacy was assessed on the Global Aesthetic Improvement Scale (GAIS). Tolerability, pain, ease of application, injection pressure were recorded.

Results: Improvement was found in $80 \%$ of patients on the GAIS, $20 \%$ being much improved. In all patients post injection tolerability was rated as „excellent“. Adverse reactions (erythema) at the injection site occurred in $33.3 \%$ of the patients. The alginate gel provided easy and rather painless injection.

Conclusion: Novabe $\mathrm{l}^{\circledR}$ is a new option for volume augmentation in up to now less frequent treated areas. 


\section{Literatur}

1 Clinical study of Novabel ${ }^{\circledR}$ in nasolabial folds; Multicenter, uncontrolled clinical study to investigate effectiveness and safety of Novabel ${ }^{\circledR}$ after bilateral mid-to-deep dermal implantation for correction of moderate to severe facial wrinkles and folds such as nasolabial folds; Interim data after 15 months, Merz Pharmaceuticals GmbH, Data on file.

2 Lemperle G, Morhenn V, Charrier U. Human histology and persistance of various injectable filler substances for soft tissue augmentation. Aesth Plast Surg 2003; 27: $354-366$

3 Narins RS, Brandt F, Leyden J et al. A randomized, double-blind, multicenter comparison of the efficacy and tolerability of Restylane ${ }^{\circledR}$ versus
Zyplast $^{\circledR}$ for the correction of nasolabial folds. Dermatol Surg 2003; 29: $588-595$

4 Pavicic T. Filler - ein Überblick. J Ästhet Chir 2009; 2: 15-22

5 Taufig AZ, Szöke A, Kühnel W. Neue Strategie zur Erfassung intradermaler Reaktionen nach Implantation resorbierbarer Dermalfiller. J Ästhet Chir 2009; 2: 29-32

6 US-Packungsbeilagen Juvederm Ultra (Juvéderm 24 HV), Juvederm Ultra Plus (Juvéderm 30 HV), Restylane (Staudy MA-1400-02), Perlane (Study MA-1400-02).

7 Zimmermann US, Clerici TJ. The histological aspects of fillers complications. Semin Cutan Med Surg 2004; 23: 241 - 250

8 Merz Pharmaceuticals, Data on file. 